DOI: 10.2478/romneu-2014-0009

\title{
Long segment dorsolumbar spinal arachnoid cyst: a case report
}

\author{
Pankaj Gupta ${ }^{1}$, Nityanand Gopal ${ }^{2}$, Varsha Kumar ${ }^{3}$ \\ ${ }^{1}$ Registrar, Department of Neurosurgery, Sawai Man Singh Medical College, Jaipur, Rajasthan - \\ 302004 \\ ${ }^{2}$ Associate Professor, P.G. Department of Surgery, M.L.N. Medical College, Allahabad, Uttar \\ Pradesh - 211001 \\ ${ }^{3}$ Senior Resident, Department of Pathology, Sanjay Gandhi Post-Graduate institute of Medical \\ Sciences, Lucknow, Uttar Pradesh - 226014
}

\section{Abstract}

$$
\text { Congenital spinal intradural }
$$
arachnoid cyst involving almost the entire spine is very rare. We report a case of 15 years old boy, who presented with progressive spastic paraparesis with gait instability for last 4 months. MRI spine revealed thoracolumbar arachnoid cyst extending from D4 to L3 segment. Complete surgical excision of arachnoid cyst and laminoplasty was done. Patient recovered completely and histopathological examination of specimen confirmed the diagnosis of arachnoid cyst.

\section{Introduction}

Arachnoid cysts are pouches of CSF, found within subarachnoid space. They are of two types, Primary Spinal intradural arachnoid cysts are uncommon congenital lesions that arise during development from the splitting of arachnoid membrane, which contains CSF like fluid. Although the exact pathogenesis is unclear but they may result from an alteration and enlargement of the arachnoid trabeculae because of valve like mechanism. Secondary Arachnoid cysts result from trauma, meningitis, arachnoiditis or may be iatrogenic following spinal anaesthesia. It may be associated with congenital meningocoele, syringomyelia, neurofibromatosis, Marfan Syndrome or corpus callosal agenesis.According to medical literature, very rarely it may run in families as an Autosomal recessive trait. (2) The most common location of arachnoid cyst in spine is thoracic segment, dorsal to the spinal cord, while in brain it is middle cranial fossa. (3) Although it is congenital in origin but most common age of presentation is between 30 to 50 years of age, with slight female predominance. $(4,5)$ Spinal arachnoid cysts almost always communicate with intrathecal subarachnoid space through a small defect in the dura. Usually they are asymptomatic and rarely they can cause spinal cord compression, 
producing local/radicular pain, paraparesis/paraplegia, sensory disturbances, gait instability/ataxia and sphincter disturbances. Rarely they may present with intracystic haemorrhage or with intracranial hypotension due to spontaneous cyst rupture. (5)

\section{Case report}

A 15 years old boy presented to the neurosurgery OPD with chief complaints of weakness, numbness of both lower limbs and instability during walking for last 4 months. The onset of symptoms was insidious and gradually progressive in nature. There was no history of trauma, spinal surgery, infection or spinal anaesthesia. On examination spastic paraparesis was present. Motor power of both lower limbs was $4 / 5$ at hip and knee and $3 / 5$ at ankle and toes. Deep tendon reflex was bilaterally brisk and Babinski's sign was present. No clinical evidence of Neurofibromatosis was present. MRI of spine revealed intradural-extramedullary, multiloculated, large, cystic lesion extending from D4 to L3 segment, which was hypointense to cord on T1W1 and hyperintense on T2W1. Lesion was isointense to CSF, showing restriction on DWI images. There was no enhancement on contrast study. The cord compression was most marked from D9 to L3 region.

Patient was operated electively and D4 to L3 segment Open Door Laminoplasty done. Dura was tense and bulging. Vertical midline durotomy done. On opening the arachnoid layer, CSF escaped out under high pressure and the cyst filled with CSF became visible. The cyst was large, multiloculated, covering the dorsal aspect of spinal cord, extending from D4 to L3 segment and was producing cord compression, which was evidenced by thinning of cord and absence of cord pulsation. The cystic membrane was exposed and resected in piecemeal. After excision of cyst the dorsal portion of the cord gradually regained its normal diameter and adequate cord pulsation returned back. Water tight dural repair was done using and no CSF leak was observed peroperatively.

The postoperative period was uneventful. Patient recovered almost completely and the power of both lower limbs was $5 / 5$ on postoperative day 4 . Patient could walk on postoperative day 8 , without gait instability. Histopathological examination of the resected specimen confirmed the diagnosis of Arachnoid cyst. On regular follow up, the boy could walk and run without difficulty. Till date patient has no complaints suggestive of recurrence of Arachnoid cyst.

\section{Discussion}

Spinal intradural Arachnoid cyst is an uncommon entity and it rarely leads to symptomatic spinal cord compression. To the best of our knowledge, Baysefer et al. (2001) has reported such a giant Arachnoid cyst in a 2 years old boy, extending from D3 to L1 segment. (2) 
DOI: 10.2478/romneu-2014-0009

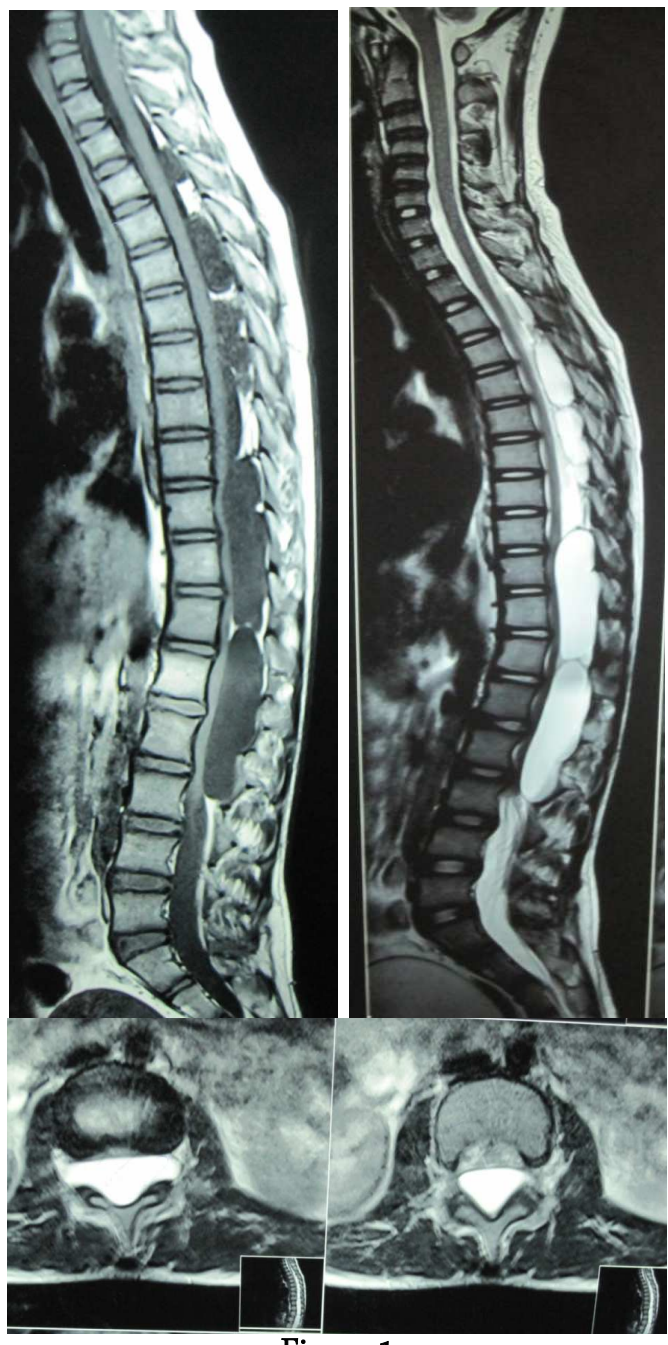

Figure 1

MRI Saggital and axial section of spine showing intradural-extramedullary, multiloculated, large, cystic lesion extending from D4 to L3 segment, which is hypointense to cord on T1W1 and hyperintense on T2W1. The lesion is isointense to CSF.

The conservative management is reserved for asymptomatic patients of spinal Arachnoid cyst, while complete neurosurgical excision is the treatment of choice in symptomatic cases. Extensively giant cysts, ventrally located cysts, or cysts densely adhered to the cord can be treated by partial resection and fenestration of the cyst, so that it can freely communicate with subarachnoid space. $(2,5)$ Other options of treatment are cystoperitoneal shunt, cystopleural shunt, cystoarachnoid shunt, marsupialisation, endoscopic/Laser fenestration and reservoir placement. (1) Simple aspiration of cyst is associated with unacceptable high recurrence rate, so it is not a recommended treatment modality. (2) The role of pharmacotherapy is limited to symptomatic relief only.

\section{Footnotes:}

Source of support: Nil

Conflicts of interest: None declared

Correspondence address:

Dr. Nityanand Gopal(MCh)

Associate Professor Neurosurgery

P.G. Department of Surgery

Swaroop Rani Nehru Hospital

Moti Lal Nehru Medical College

Allahabad Uttar Pradesh 211001.

emailid:drpankaj.gupta@yahoo.co.in,

nnggopal@yahoo.co.in

\section{References}

1. Alvisi C, Cerisoli M, Giulioni M, Guerra L; Longterm results of surgically treated congenital intradural spinal arachnoid cysts. Journal of Neurosurgery; 67(3):333-335, 1987.

2. Baysefer A, Lzci Y, Erdogan E; Lateral intrathoracic meningocele associated with a spinal intradural arachnoid cyst. Pediatric Neurosurgery; 35(2):107-110, 2001.

3. Elsberg CA, Dyke CG, Brewer ED; Bull. Neurol. Inst. N.Y., iii, 395, 1934.

4. Lee HJ, Cho DY; Symptomatic spinal intradural arachnoid cysts in the pediatric age group: description of three new cases and review of the literature. Pediatric Neurosurgery; 35(4):181-187, 2001.

5. Wang MY, Levi AD, Green BA; Intradural spinal arachnoid cysts in adult. Surgical Neurology; 60(1):4956, 2003. 